The inflating curvaton

This article has been downloaded from IOPscience. Please scroll down to see the full text article.

JCAP03(2012)022

(http://iopscience.iop.org/1475-7516/2012/03/022)

View the table of contents for this issue, or go to the journal homepage for more

Download details:

IP Address: 194.80.32.10

The article was downloaded on 12/04/2012 at 18:47

Please note that terms and conditions apply. 


\title{
The inflating curvaton
}

\section{Konstantinos Dimopoulos, ${ }^{a}$ Kazunori Kohri, ${ }^{b}$ David H. Lyth ${ }^{a}$ and Tomohiro Matsuda ${ }^{c, d}$}

\author{
${ }^{a}$ Consortium for Fundamental Physics, Department of Physics, Lancaster University, \\ Lancaster LA1 4YB, U.K. \\ ${ }^{b}$ Cosmophysics Group, Theory Center, IPNS, KEK, \\ and The Graduate University for Advanced Study (Sokendai), Tsukuba 305-0801, Japan \\ ${ }^{c}$ Department of Physics, Lancaster University, \\ Lancaster LA1 4YB, U.K. \\ ${ }^{d}$ Laboratory of Physics, Saitama Institute of Technology, \\ Fukaya, Saitama 369-0293, Japan \\ E-mail: konst.dimopoulos@lancaster.ac.uk, kohri@post.kek.jp, \\ d.lyth@lancaster.ac.uk, matsuda@sit.ac.jp
}

Received December 19, 2011

Accepted February 16, 2012

Published March 12, 2012

\begin{abstract}
The primordial curvature perturbation $\zeta$ may be generated by some curvaton field $\sigma$, which is negligible during inflation and has more or less negligible interactions until it decays. In the current scenario, the curvaton starts to oscillate while its energy density $\rho_{\sigma}$ is negligible. We explore the opposite scenario, in which $\rho_{\sigma}$ drives a few $e$-folds of inflation before the oscillation begins. In this scenario for generating $\zeta$ it is exceptionally easy to solve the $\eta$ problem; one just has to make the curvaton a string axion, with anomaly-mediated susy breaking which may soon be tested at the LHC. The observed spectral index $n$ can be obtained with a potential $V \propto \phi^{p}$ for the first inflation; $p=1$ or 2 is allowed by the current uncertainty in $n$ but the improvement in accuracy promised by Planck may rule out $p=1$. The predictions include (i) running $n^{\prime} \simeq 0.0026(0.0013)$ for $p=1(2)$ that will probably be observed, (ii) non-gaussianity parameter $f_{\mathrm{NL}} \sim-1$ that may be observed, (iii) tensor fraction $r$ is probably too small to ever observed.
\end{abstract}

Keywords: inflation, alternatives to inflation, physics of the early universe

ArXiv ePrint: 1110.2951 
Introduction. The primordial curvature perturbation $\zeta$ is already present a few Hubble times before cosmological scales start to enter the horizon. At that stage, and on those scales, its Fourier components $\zeta(\mathbf{k})$ are time-independent and set the principle (or only) initial condition the subsequent formation of large-scale structure in the Universe [1]. As a result, $\zeta(\mathbf{k})$ can be determined, and one of the main tasks of theoretical cosmology is to explore models of the early universe that can generate it.

The generation of $\zeta(\mathbf{k})$ presumably starts at horizon exit during inflation $(k=a H \equiv$ $\dot{a}$ where $a(t)$ is the scale factor of the universe) when the vacuum fluctuation of one or more scalar (or vector [2]) fields becomes a classical perturbation. According to the original scenario, $\zeta$ is generated by the perturbation $\delta \phi$ of the inflaton field in a single-field slow-roll inflation model. In that case, $\zeta$ is generated promptly at horizon exit, remaining constant thereafter. According to the curvaton scenario [3], $\zeta$ is instead generated by the perturbation $\delta \sigma$ of a 'curvaton' field, that has practically no effect during inflation and generates $\zeta$ only when its energy density becomes a significant fraction of the total.

Instead of the curvaton scenario one can consider an inflaton-curvaton scenario where both $\delta \phi$ and $\delta \sigma$ contribute significantly to $\zeta$ [4]. One can also suppose that $\zeta$ is generated during multi-field inflation, or by a 'modulating' field that causes an effective mass or coupling to be inhomogeneous [1]. In this Letter we stay with the simpler curvaton scenario.

Up till now, it has been assumed that the curvaton starts to oscillate while its energy density $\rho_{\sigma}$ is a negligible fraction of the total. Here we assume instead that $\rho_{\sigma}$ comes to dominate the total while it is still slowly varying, giving rise to a second era of inflation. For simplicity, we demand that these 'cosmological scales' are outside the horizon $(k<a H)$ when the second inflation begins.

A second inflation has been discussed many times before, but always within a scenario where the first inflation generates at least a significant part of $\zeta$, and/or some or all cosmological scales start out within the horizon. The second inflation is usually supposed to begin while the inflaton of the first inflation is still oscillating. The contribution of the second inflation for this 'double inflation' is calculated for instance in $[5,6]$ taking cosmological scales to be outside the horizon, and in [7] taking them to be partially inside. A second inflation starting during radiation domination might be called late inflation. Slow-roll late inflation was considered in [8], and fast-roll late inflation in [9], but these authors ignore the effect on $\zeta$ of the second inflation. There is also thermal inflation [10] which really has no effect on $\zeta$. Our scenario is different from all of these.

Duration of the second inflation. By 'cosmological' scales we mean those probed more or less directly by observation. They range from $k=a_{0} H_{0}$ where 0 denotes the present, to around $k=e^{15} a_{0} H_{0}$. According to a standard calculation [1], requiring that they are outside the horizon when the second inflation starts corresponds to

$$
N_{2} \lesssim 45-\ln \left(10^{-5} M_{\mathrm{P}} / H_{2}\right) / 2,
$$

where $N_{2} \equiv \Delta(\ln a)$ is the $e$-folds of expansion during the second inflation, $H_{2} \equiv \dot{a} / a$ is the Hubble parameter then and $M_{\mathrm{P}} \equiv(8 \pi G)^{-1 / 2}=2 \times 10^{18} \mathrm{GeV}$. [A subscript $1(2)$ will always denote the first (second) inflation.]

The right hand side of eq. (1) takes $\rho=3 M_{\mathrm{P}}^{2} H^{2}$ to be constant during the second inflation and then $\propto a^{-4}$ (radiation) till the observed matter-dominated era. Replacing some of the radiation domination by matter domination reduces the right hand side. The second term is positive because $H_{2}<H_{1}$ and we need $H_{1}<10^{-5} M_{\mathrm{P}}$ or the tensor perturbation with spectrum $\mathcal{P}_{h}=\left(8 / M_{\mathrm{P}}^{2}\right)\left(H_{1} / 2 \pi\right)^{2}$ would have been observed (see eq. (9) below). 
CDM and baryon number cannot be created before the curvaton (or any other mechanism) creates $\zeta$, since that would give an isocurvature perturbation excluded by observation [3]. That requires something like $\rho_{2}^{1 / 4}>10^{3} \mathrm{GeV}$ corresponding to $H_{2}>10^{-30} M_{\mathrm{P}}$ and $N_{2}<16$. We therefore require roughly $N_{2}<45$ to 16 .

Calculating the curvature perturbation. The curvature perturbation $\zeta$ is described non-perturbatively through the $\delta N$ formalism as in [11]. In this paper, we just work to first order in $\delta \rho$, as in [3]. Then

$$
\zeta(\mathbf{k}, t)=-H(t) \frac{\delta \rho(\mathbf{k}, t)}{\dot{\rho}(t)}=\frac{1}{3} \frac{\delta \rho(\mathbf{k}, t)}{\rho(t)+p(t)},
$$

where $p$ is the pressure, and $\delta \rho$ is defined on the slicing with uniform locally-defined scale factor $a(\mathbf{x}, t)$ (flat slicing). The second equality corresponds to the energy continuity equation $\dot{\rho}=-3 H(\rho+p)$.

Keeping only super-horizon scales, the energy continuity equation is valid locally. As a result [12], $\zeta$ is constant during any era when $p(\mathbf{x}, t)$ is a unique function of $\rho(\mathbf{x}, t)$; that is the case for pure radiation $(p=\rho / 3)$ or matter $(p=0)$. When cosmological scales start to enter the horizon, the temperature $T$ is somewhat less than $1 \mathrm{MeV}$ and we know that the Universe is practically pure radiation giving a time-independent $\zeta(\mathbf{k}, t)$ that we are denoting simply by $\zeta(\mathbf{k})$. According to the curvaton scenario, $\zeta(\mathbf{k}, t)$ does not vary between curvaton decay at $t=t_{\mathrm{d}}$ and $T \sim \mathrm{MeV}$, which is guaranteed if the universe is completely radiation-dominated throughout that era.

In any curvaton scenario, $\zeta$ is generated while $\rho=\rho_{\sigma}+\rho_{\mathrm{r}}$ and $p=p_{\sigma}+p_{\mathrm{r}}$, where $\rho_{\sigma}=V(\sigma)+\dot{\sigma}^{2} / 2$ and $p_{\sigma}=-V(\sigma)+\dot{\sigma}^{2} / 2$ are the curvaton contributions. For the original curvaton $\rho_{\mathrm{r}}$ cannot be matter and is taken to be pure radiation, $\rho_{\mathrm{r}} \propto a^{-4}$. For the inflating curvaton, $\rho_{\mathrm{r}}$ might be matter, $\rho_{\mathrm{r}} \propto a^{-3}$ allowing it to correspond to the oscillation of a field $\phi$ responsible for the first inflation. It might even decrease more slowly, say like $a^{-2}$ corresponding to a frustrated cosmic string network, although it is generally very difficult to achieve frustration for cosmic strings [13].

In these cases though, we demand that $\rho_{\mathrm{r}}$ becomes radiation before it is a significant fraction of $\rho$ after the curvaton inflation, so that $\rho_{r}$ does not cause $\zeta$ to vary significantly.

To facilitate an analytic calculation, one writes eq. (2) as $\zeta(\mathbf{k}, t)=f(t) \zeta_{\sigma}(\mathbf{k}, t)$ where $3 \zeta_{\sigma} \equiv\left(\delta \rho_{\sigma}\right) /\left(\rho_{\sigma}+p_{\sigma}\right)$ and $f(t) \equiv\left(\rho_{\sigma}+p_{\sigma}\right) /(\rho+p)$. There is supposed to be negligible exchange of energy between the two components, so that $\zeta_{\sigma}$ is constant if $p_{\sigma}(\mathbf{x}, t)$ is a unique function of $\rho_{\sigma}(\mathbf{x}, t)$. For the original curvaton, $\zeta_{\sigma}$ becomes constant only after the oscillation begins, when $p_{\sigma} \simeq 0$. We now argue that for the inflating curvaton, $\zeta_{\sigma}$ will become constant soon after the second inflation begins at the epoch $t=t_{2}$. We begin with the following equation, valid in the absence of perturbations [1]:

$$
\ddot{\sigma}+3 H \dot{\sigma}+V^{\prime}(\sigma)=0 .
$$

Since sub-horizon modes of $\sigma(\mathbf{x}, t)$ redshift away that quantity has negligible spatial gradient [1]. As a result it satisfies eq. (3) for each $\mathbf{x}$, with $3 M_{\mathrm{P}}^{2} H^{2}=\rho_{\sigma}+\rho_{\mathrm{r}}$ the locally defined quantity and $t$ the proper time. Also, $p_{\sigma}$ is a unique function of $\rho_{\sigma}$ if and only if $\sigma(\mathbf{x}, t)$ is unique up to the choice of $t=0$ (attractor solution). We expect that soon after the inflation begins it will be slow-roll or fast-roll (see below), and both of these make $\dot{\sigma}$ a unique function of $\sigma$ giving indeed the required attractor solution. 
If $\rho_{\mathrm{r}}$ were completely negligible we could invoke a more general argument for the attractor [14], but that might not apply because although the contribution of $\rho_{\mathrm{r}}$ to $H$ becomes small soon after the second inflation begins, its contribution to

$$
2 \epsilon_{H} \equiv 2|\dot{H}| / H^{2}=3\left(\dot{\sigma}^{2}+\frac{4}{3} \rho_{\mathrm{r}}\right) / \rho
$$

may be dominant at least initially. While that is happening, $f(t) \simeq\left(\dot{\sigma}^{2}\right) /\left(4 \rho_{\mathrm{r}} / 3\right) \ll 1$. But just before $\sigma$ decays at $t_{\mathrm{d}}$ we have $f\left(t_{\mathrm{d}}\right) \simeq \rho_{\sigma} /\left[\rho_{\sigma}+(4 / 3) \rho_{\mathrm{r}}\right]$ which will be very close to 1 , In contrast, the oscillating curvaton can have $f\left(t_{\mathrm{d}}\right) \ll 1$.

Keeping only super-horizon modes, $f\left(t_{\mathrm{d}}\right)=1$ gives $\zeta(\mathbf{x})=\delta \rho_{\sigma}\left(\mathbf{x}, t_{2}\right) / 3 \dot{\sigma}^{2}\left(t_{2}\right)$. To first order in $\delta \sigma, \zeta(\mathbf{x})=V^{\prime} \delta \sigma(\mathbf{x}) / 3 \dot{\sigma}^{2}$. At horizon exit during the first inflation $\delta \sigma$ is nearly gaussian with spectrum $H_{1} / 2 \pi$. Allowing $\sigma\left(\mathbf{x}, t_{2}\right)$ to be a function $g$ of its value at horizon exit, but taking both $\sigma(\mathbf{x}, t)$ and $H_{1}$ to be time-independent while cosmological scales leave the horizon, we get the scale-independent spectrum

$$
\mathcal{P}_{\zeta}^{1 / 2} \simeq \frac{g^{\prime}}{3} \frac{V^{\prime}\left(\sigma\left(t_{2}\right)\right)}{\dot{\sigma}^{2}\left(t_{2}\right)} \frac{H_{1}}{2 \pi} .
$$

Observation gives [15] $\mathcal{P}_{\zeta}^{1 / 2} \simeq 5 \times 10^{-5}$. This formula is obtained taking $g$ to be linear in $\sigma\left(t_{1}\right)$.

Taking into account the time-dependence of $H_{1}$ and $\sigma$ while cosmological scales leave the horizon one finds [1]

$$
n(k)-1 \equiv d \ln \mathcal{P} / d \ln k=-2 \epsilon_{H 1}+2 \eta_{1},
$$

with the right hand side evaluated at horizon exit $k=a H$ and $3 H^{2} \eta \equiv \partial^{2} V / \partial \sigma^{2}$. [Note that $V=V(\sigma, \phi, \cdots)$ during the first inflation where $\phi, \cdots$ are the fields responsible for that inflation.] Assuming a tensor fraction $r \equiv \mathcal{P}_{h} / \mathcal{P}_{\zeta} \ll 10^{-1}$ and $\left|n^{\prime}\right| \ll 10^{-1}$ where $n^{\prime} \equiv d n / \ln k$, observation [15] gives $n-1=-0.037 \pm 0.014$.

Eq. (6) is a universal formula, applying whenever a field $\sigma$ different from the inflaton generates $\zeta$. It can easily happen (as in our case, see below) that the last term is negligible. Then we need $2 \epsilon_{H 1} \simeq 0.037$, leading to three important consequences.

1. To get the required $\epsilon_{H 1}$ we need [16] a large change in the inflaton field $\phi$ during the first inflation. To achieve that one usually takes $\phi$ to have the canonical kinetic term with $V(\phi, \sigma) \simeq V(\phi)=A \phi^{p}$. Then $\epsilon_{H 1} \simeq p / 4 N(k)$, where $N(k)$ is the number of $e$-folds of the first inflation after the scale $k$ leaves the horizon [1]. Defining $N_{1} \equiv N\left(a_{0} H_{0}\right)$, we need $N_{1} \simeq 14 p$ to get the required $2 \epsilon_{H 1} \simeq 0.037$. While one is free to postulate any $p$ the only choices of $p$ with good justification are $p=1$ (corresponding to monodromy [17]) and $p=2$ (corresponding to 'extranatural' [18] inflation or $N$-flation [19]. These give $N_{1} \simeq 14$ and 28 respectively.

2. A standard calculation [1] gives $N_{1} \simeq 60-\ln \left(10^{-5} M_{\mathrm{P}} / H_{1}\right) / 2-N_{2}$, where the equality would be exact if $\rho$ were constant during both inflations and $\propto a^{-4}$ otherwise until the observed matter-dominated era. Combining this with eq. (1) gives

$$
15+\frac{1}{2} \ln \left(H_{1} / H_{2}\right) \lesssim N_{1} \lesssim 60-\frac{1}{2} \ln \left(10^{-5} M_{\mathrm{P}} / H_{1}\right) .
$$

Taking into account the uncertainty in $n$ this is compatible with $N \simeq 14 p$ for $p=1$ or 2 , though $p=1$ may be ruled out when Planck reduces the uncertainty. For the oscillating curvaton, where $N_{2}$ is absent, we would probably need $p \sim 3$ to 4 . 
3. Since $n(k)-1 \propto 1 / N(k)$ the 'running' $n^{\prime}$ is given by $n^{\prime}=(1-n) / N_{1}$. This prediction holds also for the oscillating curvaton scenario (if $\left|\eta_{1}\right| \ll \epsilon_{H 1}$ ), and for the inflaton scenario within some simple slow-roll models and it makes $n^{\prime}$ big enough to observe in the future. For the oscillating curvaton, and the inflaton scenario, one expects roughly $N_{1} \simeq 60$ corresponding to $n^{\prime} \simeq 0.0007$. For the inflating curvaton, we have for $V(\phi) \propto \phi^{p}$ and taking account of the uncertainty in $n$

$$
n^{\prime}=\left(\frac{1-n}{0.037}\right)^{2} \frac{0.0026}{p}
$$

with $p=1$ or 2 . This precise prediction for $n^{\prime}$ will be probably be tested in the future (see for instance [20]).

We require the contribution of the first inflation to be negligible, $s \equiv \mathcal{P}_{\zeta_{\phi}} / \mathcal{P}_{\zeta} \ll 1$. Assuming canonical kinetic terms for the inflaton(s) [1],

$$
\mathcal{P}_{\zeta_{\phi}}^{1 / 2} \geq \frac{1}{\sqrt{2 \epsilon_{H 1}}} \frac{H_{1}}{2 \pi M_{\mathrm{P}}}
$$

and $r \leq 16 s \epsilon_{H 1}$ implying $H_{1} \leq 1.1 \times 10^{-4}(r s)^{1 / 2} M_{\mathrm{P}}$. (The equalities hold for a single inflaton.) Observation [15] gives $r \lesssim 10^{-1}$. Even if the second term of eq. (6) contributes significantly, it is unlikely to accurately cancel the first term which means that we need $\epsilon_{H 1} \lesssim 0.02$, giving $r \lesssim 0.3 s$ and $H_{1} \lesssim 6 \times 10^{-5} s^{1 / 2} M_{\mathrm{P}}$. The tensor fraction will not be observed by Planck [21] if $s \lesssim 10^{-1}$, and it will probably never be observed [22] if $s \lesssim 10^{-3}$.

To calculate the non-gaussianity parameter $f_{\mathrm{NL}}$ we expand $\delta \rho_{\sigma}$ to second order in $\delta \sigma$ giving

$$
\begin{aligned}
\zeta(\mathbf{x}) & =V^{\prime} \delta \sigma / 3 \dot{\sigma}^{2}+(3 / 5) f_{\mathrm{NL}}\left(V^{\prime} \delta \sigma / 3 \dot{\sigma}\right)^{2} \\
f_{\mathrm{NL}} & =\left(5 \dot{\sigma}^{2} V^{\prime \prime} / V^{\prime 2}\right)\left(1+g^{\prime \prime} / g^{\prime 2}\right) .
\end{aligned}
$$

Observation [15] requires $-10<f_{\mathrm{NL}}<74$ which means that the second term of eq. (10) gives a negligible contribution [23] to $\mathcal{P}_{\zeta}$. We need $\left|f_{\mathrm{NL}}\right| \gtrsim 1$ if $f_{\mathrm{NL}}$ is ever to be detected [24]. With such a value it will indeed be a good approximation to ignore the non-gaussianity of $\delta \sigma$ [25]. But our first-order treatment of $\delta \rho_{\sigma}$ is reliable only [1] for $\left|f_{\mathrm{NL}}\right| \gg 1$; for $\left|f_{\mathrm{NL}}\right| \sim 1$ one should go to second order, or use the $\delta N$ formalism as in [3].

The usual way of achieving inflation would be through the slow roll approximation $\dot{\sigma} \simeq-V^{\prime} / 3 H_{2}$. That will typically make $N_{2}$ too large but let us anyway see what it implies. Differentiating it requires $\epsilon_{H 2} \ll 1$ and $\left|\eta_{2}\right| \ll 1$ where $\eta_{2} \equiv V^{\prime \prime} / 3 H_{2}^{2}$. Using eq. (4) the former condition requires $\epsilon_{2} \equiv M_{\mathrm{P}}^{2}\left(V^{\prime} / V\right)^{2} / 2 \ll 1$ and $\rho_{\mathrm{r}} \ll \rho$. Then we get

$$
\begin{aligned}
\mathcal{P}_{\zeta}^{1 / 2} & =\left(2 \epsilon_{2}\right)^{-1 / 2}\left(H_{1} / 2 \pi\right)\left(g^{\prime} / M_{\mathrm{P}}\right) \\
(3 / 5) f_{\mathrm{NL}} & =\eta_{2}\left(1+g^{\prime \prime} / g^{\prime 2}\right) .
\end{aligned}
$$

We would need $\left|g^{\prime \prime}\right| \gg g^{\prime 2}$ to get a detectable $f_{\mathrm{NL}}$.

Most discussions of the oscillating curvaton take $V(\sigma) \simeq m_{\sigma}^{2} \sigma^{2} / 2$. This choice is impossible for the inflating curvaton scenario if the first inflation has inflaton(s) with canonical kinetic terms. Indeed, using eqs. (9) and (13) the curvaton contribution $\mathcal{P}_{\zeta_{\sigma}}$ is given by $\mathcal{P}_{\zeta_{\sigma}} / \mathcal{P}_{\zeta_{\phi}}=2 \epsilon_{H 1} N_{2}\left(\sigma_{2} / \sigma_{1}\right)^{2} \lesssim 1$ which means that $\mathcal{P}_{\zeta_{\sigma}}$ cannot dominate. 
The curvaton a string axion. Any scheme for generating $\zeta$ from some field $\chi$ encounters the $\eta$ problem; that a generic supergravity theory gives [26] in the early universe $\left|\eta_{\chi}^{\mathrm{nr}}\right| \gtrsim 1$, where $\eta_{\chi}^{\mathrm{nr}} \equiv\left(\partial^{2} V / \partial \chi^{2}\right) / 3 H_{\mathrm{nr}}^{2}$ and $3 M_{\mathrm{P}}^{2} H_{\mathrm{nr}}^{2} \equiv \rho_{\mathrm{nr}}$ excludes any radiation contribution. It is a problem for two reasons; (i) we need $\left|\eta_{\chi}\right| \lesssim 10^{-2}$ while cosmological scales leave the horizon to keep $|n-1|$ small enough, (ii) unless $|\partial V / \partial \chi|$ is exceptionally small we generally need $\left|\eta_{\chi}\right| \ll 1$ at all times or $\chi$ will be quickly driven to a minimum of $V$.

For the inflaton scenario, the $\eta$ problem exists only during inflation when $H \simeq$ const; it is often ignored and could be regarded as a fine-tuning requirement on the parameters of the supergravity inflaton potential. For the oscillating curvaton scenario the $\eta$ problem may be more severe because it may exist for a long time after inflation, with $H$ strongly decreasing. For the inflating curvaton it is definitely more severe because it exists during both inflations with very different values for $H$, in that the generic value $|\eta| \sim 1$ would imply a very strong evolution of $\partial^{2} V / \partial \sigma^{2}$ between the two evolutions corresponding to a highly non-trivial potential $V(\sigma, \phi, \ldots)$.

To avoid the $\eta$ problem for the curvaton one can take it be a pNGB with the potential

$$
V(\sigma)=\frac{1}{2} V_{0}\left[1+\cos \left(\frac{\pi \sigma}{\sigma_{0}}\right)\right]
$$

practically independent of other field values. It is known [3] that $\sigma_{0} \ll M_{\mathrm{P}}$ gives the oscillating curvaton, but it is unclear how to motivate such a value. Choosing instead $\sigma_{0} \gg M_{\mathrm{P}}$ would give a second inflation with $\left|\eta_{2}\right| \ll 1$, but $N_{2}$ would typically be too big and this choice anyhow seems impossible within string theory [27]. (If the latter difficulty is ignored we can replace $\sigma$ in eq. (14) by $\phi$ to arrive at 'Natural Inflation' [1].)

What we need for the inflating curvaton is $\sigma_{0} \sim M_{\mathrm{P}}$. Then, in the regime $\sigma \ll \sigma_{0}$ we have $V \simeq V_{0}-m^{2} \sigma^{2} / 2$ giving $\left|\eta_{2}\right| \sim 1$ and just a few $e$-folds of inflation. Setting $3 M_{\mathrm{P}}^{2} H_{2}^{2}=V_{0}$ gives $H_{2}^{2} / m^{2} \simeq\left(2 / 3 \pi^{2}\right)\left(\sigma_{0}^{2} / M_{\mathrm{P}}^{2}\right)$. The required value $\sigma_{0} \sim M_{\mathrm{P}}$ is achieved if $\sigma$ is a string axion with gravity- or anomaly mediated susy breaking. [27]. Then $m$ is of order the gravitino mass $m_{g}[27]$. The curvaton and gravitino have to decay before they can upset BBN which requires [28] $m \gtrsim 10^{4} \mathrm{GeV} \sim 10^{-14} M_{\mathrm{P}}$. This corresponds to anomaly mediation, which gives $m \sim 10^{4}$ to $10^{5} \mathrm{GeV}$ and (like any version of susy) may soon be tested at the LHC.

The contribution of $\eta_{1}$ to $n-1$ will be negligible if $H_{1} / m \gg[3(1-n) / 2]^{-1 / 2}=4$. With such a low $m$ this is a mild requirement which we will take to be satisfied, so that eq. (8) holds.

Writing $V^{\prime}=-m^{2} \sigma$, and taking $H_{2}$ to be constant, eq. (3) gives during the second inflation $[29]$

$$
\dot{\sigma} \simeq F H_{2} \sigma, \quad F \equiv-\frac{3}{2}+\sqrt{\frac{9}{4}+\frac{m^{2}}{H_{2}^{2}}} \sim m / H_{2} .
$$

The slow-roll regime is $m \ll H_{2}$ but we are interested in the 'fast-roll' [29] regime $m \gtrsim H_{2}$ corresponding to $F \gtrsim 1$. (The approximation $F \simeq m / H_{2}$ is adequate for $m^{2} / H_{2}^{2} \gtrsim 3$.) In the fast-roll regime, eq. (15) is self-consistent if $N_{2} \gg 1$ and $\sigma \ll \sigma_{0}$.

Since eq. (3) is linear, $g$ is a linear function and we get

$$
\begin{aligned}
\mathcal{P}_{\zeta}^{1 / 2} & =\frac{1}{3}\left(\frac{m}{F H_{2}}\right)^{2} \frac{H_{1}}{2 \pi \sigma_{1}} \sim \frac{H_{1}}{6 \pi \sigma_{1}} \\
(3 / 5) f_{\mathrm{NL}} & =-\left(F H_{2} / m\right)^{2} \sim-1 .
\end{aligned}
$$


The result for $f_{\mathrm{NL}}$ may be strongly modified by the correction of second order in $\delta \rho_{\sigma}$, but barring a strong cancellation it seems that $f_{\mathrm{NL}}$ may eventually be detectable.

Since inflation ends at $\sigma \sim \sigma_{0}$ we have $F N_{2} \sim \ln \left(M_{\mathrm{P}} / \sigma_{2}\right)$. Since $N_{2} \gg 1$, we have $F \ll F N_{2}$, hence $m / H_{2} \ll F N_{2}$. Using eq. (16) with $H_{1}>H_{2} \gtrsim 10^{4} \mathrm{GeV}$ gives $F N_{2} \lesssim 24$. Going the other way, eq. (16) with $H_{1} \sim 10^{-6} M_{\mathrm{P}}$ implies $F N_{2} \lesssim 4$.

Eqs. (16) and (17) are roughly the same as those of the oscillating curvaton model with $f\left(t_{\mathrm{d}}\right) \simeq 1$. But the result for $n^{\prime}$ is different for the oscillating curvaton [1]; there we might have $n-1 \simeq 2 \eta_{1}$ with $n^{\prime}$ negligible, and even if eq. (8) holds we expect $p \simeq 4$.

One may worry about the assumption that $\sigma\left(t_{1}\right)$ is near the top of the potential, given that the first inflation may be of long duration. For a given $H_{1}(t)$, the late-time probability distribution of $\sigma$ at the end of the first inflation can be calculated [30]. Taking that distribution to apply and also taking $H_{1}$ to be constant, we would need $H_{1}^{4} \gtrsim V_{0}$ to have a significant probability that $\sigma$ is near the top. This requires $H_{1} / H_{2} \gtrsim M_{\mathrm{P}} / H_{1} \gtrsim 10^{5}$ and (since $H_{2}>10^{4} \mathrm{GeV}$ ) $H_{1} \gtrsim 10^{-7} M_{\mathrm{P}}$. The former bound would probably make eq. (7) incompatible with $p=1$. But to know whether the estimate $H_{1}^{4} \gtrsim V_{0}$ is realistic one would have to calculate the evolution of the probability distribution with the correct $H_{1}(t)$ and a range of initial values of $\sigma$.

Conclusion. The hypothesis that the curvaton is a string axion leads to a simple earlyuniverse scenario. The curvaton generates a few $e$-folds of inflation with $H \sim 10^{4} \mathrm{GeV}$, during which $\zeta$ is created. The main inflation takes place earlier, with a potential $V(\phi) \propto \phi^{p}$, with $p=1$ or 2 needed to reproduce the observed spectral index within current observational uncertainity. The hypothesis requires low-energy susy with anomaly-mediated susy breaking which may soon be tested at the LHC. It predicts that a tensor fraction $r$ that is probably too small ever to observe, but a running $n^{\prime}$ that eventually be observed and will decide between the linear and quadratic potentials. A third prediction $f_{\mathrm{NL}} \sim-1$ may also be testable, but the accuracy of the calculation needs to be improved.

\section{Acknowledgments}

TM and KK thank Anupam Mazumdar for valuable discussions in the early stage of this work. DHL thanks Michael Dine for correspondence, and has support from UNILHC23792, European Research and Training Network (RTN) grant. KD and DHL are supported (in part) by the Lancaster-Manchester-Sheffield Consortium for Fundamental Physics under STFC grant ST/J000418/1. KK is partly supported by the Grant-in-Aid for the Ministry of Education, Culture, Sports, Science and Technology, Government of Japan Nos. 21111006, 22244030, 23540327, and by the Center for the Promotion of Integrated Science (CPIS) of Sokendai (1HB5806020).

\section{References}

[1] D.H. Lyth and A.R. Liddle, The primordial density perturbation: cosmology, inflation and the origin of structure, Cambridge University Press, Cambridge U.K. (2009) [INSPIRE].

[2] K. Dimopoulos, M. Karciauskas, D.H. Lyth and Y. Rodriguez, Statistical anisotropy of the curvature perturbation from vector field perturbations, JCAP 05 (2009) 013 [arXiv:0809.1055] [INSPIRE].

[3] D.H. Lyth and D. Wands, Generating the curvature perturbation without an inflaton, Phys. Lett. B 524 (2002) 5 [hep-ph/0110002] [INSPIRE]; 
D.H. Lyth, C. Ungarelli and D. Wands, The primordial density perturbation in the curvaton scenario, Phys. Rev. D 67 (2003) 023503 [astro-ph/0208055] [INSPIRE];

see also K. Enqvist and M.S. Sloth, Adiabatic CMB perturbations in pre-big bang string cosmology, Nucl. Phys. B 626 (2002) 395 [hep-ph/0109214] [INSPIRE].

[4] A.D. Linde and V.F. Mukhanov, Non-Gaussian isocurvature perturbations from inflation, Phys. Rev. D 56 (1997) R535 [astro-ph/9610219] [InSPIRE];

T. Moroi and T. Takahashi, Effects of cosmological moduli fields on cosmic microwave background, Phys. Lett. B 522 (2001) 215 [Erratum ibid. B 539 (2002) 303] [hep-ph/0110096] [INSPIRE].

[5] D. Polarski and A.A. Starobinsky, Spectra of perturbations produced by double inflation with an intermediate matter dominated stage, Nucl. Phys. B 385 (1992) 623 [INSPIRE].

[6] K. Ichikawa, T. Suyama, T. Takahashi and M. Yamaguchi, Non-Gaussianity, spectral index and tensor modes in mixed inflaton and curvaton models, Phys. Rev. D 78 (2008) 023513 [arXiv:0802.4138] [INSPIRE].

[7] R. Allahverdi, A. Mazumdar and T. Multamaki, Large tensor-to-scalar ratio and low scale inflation, arXiv:0712.2031 [INSPIRE].

[8] L. Randall and S.D. Thomas, Solving the cosmological moduli problem with weak scale inflation, Nucl. Phys. B 449 (1995) 229 [hep-ph/9407248] [INSPIRE].

[9] G. Lazarides and C. Pallis, Reducing the spectral index in F-term hybrid inflation through a complementary modular inflation, Phys. Lett. B 651 (2007) 216 [hep-ph/0702260] [INSPIRE].

[10] D.H. Lyth and E.D. Stewart, Thermal inflation and the moduli problem, Phys. Rev. D 53 (1996) 1784 [hep-ph/9510204] [INSPIRE].

[11] D.H. Lyth and Y. Rodriguez, The inflationary prediction for primordial non-Gaussianity, Phys. Rev. Lett. 95 (2005) 121302 [astro-ph/0504045] [INSPIRE].

[12] D. Wands, K.A. Malik, D.H. Lyth and A.R. Liddle, A new approach to the evolution of cosmological perturbations on large scales, Phys. Rev. D 62 (2000) 043527 [astro-ph/0003278] [INSPIRE].

[13] C.P. Burgess, R. Easther, A. Mazumdar, D.F. Mota and T. Multamaki, Multiple inflation, cosmic string networks and the string landscape, JHEP 05 (2005) 067 [hep-th/0501125] [INSPIRE].

[14] D.S. Salopek and J.R. Bond, Nonlinear evolution of long wavelength metric fluctuations in inflationary models, Phys. Rev. D 42 (1990) 3936 [INSPIRE].

[15] WMAP collaboration, E. Komatsu et al., Seven-year Wilkinson Microwave Anisotropy Probe (WMAP) observations: cosmological interpretation, Astrophys. J. Suppl. 192 (2011) 18 [arXiv: 1001.4538] [INSPIRE].

[16] D.H. Lyth, What would we learn by detecting a gravitational wave signal in the cosmic microwave background anisotropy?, Phys. Rev. Lett. 78 (1997) 1861 [hep-ph/9606387] [INSPIRE].

[17] E. Silverstein and A. Westphal, Monodromy in the CMB: gravity waves and string inflation, Phys. Rev. D 78 (2008) 106003 [arXiv:0803.3085] [InSPIRE].

[18] N. Arkani-Hamed, H.-C. Cheng, P. Creminelli and L. Randall, Extra natural inflation, Phys. Rev. Lett. 90 (2003) 221302 [hep-th/0301218] [INSPIRE].

[19] A.R. Liddle, A. Mazumdar and F.E. Schunck, Assisted inflation, Phys. Rev. D 58 (1998) 061301 [astro-ph/9804177] [INSPIRE]; S. Dimopoulos, S. Kachru, J. McGreevy and J.G. Wacker, N-flation, JCAP 08 (2008) 003 [hep-th/0507205] [INSPIRE]. 
[20] BigBoss Experiment collaboration, D. Schelgel et al., The BigBOSS experiment, FERMILAB-FN-0932-AE-CD (2011) [INSPIRE].

[21] L.P.L. Colombo, E. Pierpaoli and J.R. Pritchard, Cosmological parameters after WMAP5: forecasts for Planck and future galaxy surveys, Mon. Not. Roy. Astron. Soc. 398 (2009) 1621 [arXiv:0811.2622] [INSPIRE].

[22] M. Hazumi, Jumping into CMB polarization measurements: a new group at KEK, AIP Conf. Proc. 1040 (2008) 78 [inSPIRE].

[23] L. Boubekeur and D.H. Lyth, Detecting a small perturbation through its non-Gaussianity, Phys. Rev. D 73 (2006) 021301 [astro-ph/0504046] [INSPIRE].

[24] V. Desjacques and U. Seljak, Primordial non-Gaussianity in the large scale structure of the universe, arXiv:1006.4763 [INSPIRE].

[25] F. Vernizzi and D. Wands, Non-Gaussianities in two-field inflation, JCAP 05 (2006) 019 [astro-ph/0603799] [INSPIRE].

[26] D.H. Lyth and T. Moroi, The masses of weakly coupled scalar fields in the early universe, JHEP 05 (2004) 004 [hep-ph/0402174] [INSPIRE].

[27] M. Dine, G. Festuccia, J. Kehayias and W. Wu, Axions in the landscape and string theory, JHEP 01 (2011) 012 [arXiv:1010.4803] [INSPIRE].

[28] M. Kawasaki, K. Kohri and T. Moroi, Big-bang nucleosynthesis and hadronic decay of long-lived massive particles, Phys. Rev. D 71 (2005) 083502 [astro-ph/0408426] [INSPIRE].

[29] A.D. Linde, Fast roll inflation, JHEP 11 (2001) 052 [hep-th/0110195] [INSPIRE].

[30] A.A. Starobinsky and J. Yokoyama, Equilibrium state of a selfinteracting scalar field in the de Sitter background, Phys. Rev. D 50 (1994) 6357 [astro-ph/9407016] [INSPIRE]. 